\title{
DYNAMO DRIVEN BY WEAK PLASMa TURBULENCE
}

\author{
Y. TONG ${ }^{1,3)}$ AND A.C.-L. CHIAN ${ }^{2,3)}$ \\ 1) Bei.jing Normal University, China \\ 2) DAMTP-University of Cambridge, UK \\ 3) National Institute for Space Research, Brazil
}

We discuss a dynamo mechanism driven by weak plasma turbulence and show that turbulent plasma waves may generate and maintain cosmic magnetic field. A dynamo equation is derived from the magnetic induction equation based on mean field electrodynamics. In the usual $\alpha-\omega$ dynamo theory, the source term in the dynamo equation arises from $\alpha$-effect associated with the convective motion of the fluid. In contrast, in our theory the source term is determined by "P-effect" associated with weakly turbulent waves (c.g. Alfvén waves) in the plasma. We suggest that "P- $\omega$ " dynamo may be operative either in the presence or absence of convection. The sole requirement for its operation is the existence of weak plasma turbulence in the source region of the cosmic magnetic field.

Using Fourier transform to express the fluactuating terms in the magnetic induction equation based on mean field clectrodynamics, we obtain a dynamo equation in the cylindrical polar coordinates $(\mathrm{R}, \varphi, \mathrm{z})$ :

$$
\frac{\partial \vec{B}_{0}}{\partial t}=\eta V^{2} \vec{B}_{0}-\left(\vec{v}_{0} \cdot V\right) \vec{B}_{0}+P \frac{\vec{B}_{0}}{1-\beta \omega R}
$$

where $P=-\frac{2}{\omega_{K}} \frac{\partial}{\partial z}\left(K_{Z}\left|U_{T}\right|^{2}\right)=$ effect of weak plasma turbulence

and $\left|U_{T}\right|^{2}=\int\left|v_{K}\right|^{2} d K=$ square of turbulent wave amplitude,

$\vec{B}_{0}=$ mean magnetic field,

$\vec{v}_{0}=$ mean fluid velocity,

$\omega=$ angular velocity of rotation,

$\beta=K_{\varphi} / \omega_{K}$,

$K_{\varphi}=\varphi$-component of wave number,

$\omega_{K}=$ dispersion frequency of turbulent plasma wave. 
Using separation of variables $\vec{B}_{0}(\vec{r}, t)=A(t) \vec{B}(\vec{r})=A_{0} e^{\lambda t} \vec{B}(\vec{r})$, Eq.(1) becomes

$$
V^{2} \vec{B}(\vec{r})-\frac{1}{\eta}(\vec{v} \cdot V) \vec{B}(\vec{r})-\frac{\lambda}{\eta} \vec{B}(\vec{r})+P \frac{\vec{B}(\vec{r})}{\eta(1-\beta \omega R)}=0
$$

Assuming axisymmetry the 3 components of Eq.(2) give

$$
\begin{gathered}
\left(V^{2}-R^{-2}\right) B_{\varphi}(R, z)-\frac{1}{\eta} B_{\varphi}(R, z)-\frac{1}{\eta} \omega(R, z) B_{R}+P \frac{B_{\varphi}}{\eta(1-\beta \omega R)}=0 \\
\left(V^{2}-R^{-2}\right) B_{R}(R, z)-\frac{\lambda}{\eta} B_{R}(R, z)+\frac{1}{\eta} \omega(R, z) B_{\varphi}+P \frac{B_{R}}{\eta(1-\beta \omega R)}=0 \\
V^{2} B_{z}(R, z)-\frac{\lambda}{\eta} B_{z}(R, z)+P \frac{B_{z}}{\eta(1-\beta \omega R)}=0
\end{gathered}
$$

Eq.(3) describes the toroidal ficld, Eqs.(4) and (5) combine to form the poloidal field.

In the non-rotation case $(\omega=0)$, Eq.(1) reduces to the dynamo equation originally derived by $\mathrm{Li}$ and Song (1981)

$$
\frac{\partial \vec{B}_{0}}{\partial t}=\eta \nabla^{2} \vec{B}_{0}+P \vec{B}_{0}
$$

which yields solutions with only the poloidal field. In the rotation case $(\omega \neq 0)$, Eqs.(3)-(5) show that the toroidal ficld can be gencrated from the poloidal field even when the rotation is constant $(\omega \neq \omega(\varphi))$. Tong et al (1985) obtained the dynamo solutions of Eqs.(3)-(5) by neglecting the third terms in Eqs.(3)-(4), thus their results apply only for very slow rotation. We have now improved the dynamo solutions of Tong ct al (1985) by considering all the terms in Eqs.(3)-(5). The resulting solutions apply to a much wider range of rotating celcstial bodies.

Li. X-Q \& Song. G-X. Astrophys. Sp. Sci.76, 13(1981).

Tong Y, Lu S-Z, Mao X-J \& Han J-L. Astrophys. Sp. Sci.113, 303(1985). 